(C) 2016 IEEE. Personal use of this material is permitted. Permission from IEEE must be obtained for all other uses, in any current or future media, including reprinting/republishing this material for advertising or promotional purposes, creating new collective works, for resale or redistribution to servers or lists, or reuse of any copyrighted component of this work in other works. 


\title{
Selecting the Optimal Movement Subset with Different Pattern Recognition Based EMG Control Algorithms
}

\author{
Ali H. Al-Timemy, Rami N. Khushaba, Senior Member, IEEE, and Javier Escudero*, Member, IEEE
}

\begin{abstract}
Pattern Recognition (PR)-based EMG controllers of multi-functional upper-limb prostheses have been recently deployed on commercial state-of-the-art prostheses, offering intuitive control with the ability to control large number of movements with fast reaction time. Current challenges with such PR systems include the lack of training and deployment protocols that can help optimize the system's performance based on amputees' needs. Selecting the best subset of movements that each individual amputee can perform will help to exclude movements that have poor performance so that a subject-specific training can be achieved. In this paper, we propose to select the best set of movements that each amputee can perform as well as identifying the movements for which the PR system would have the worst performance and, therefore, would require further training. Unlike previous studies in this direction, different feature extraction and classification methods were utilized to examine if the choice of features/classifiers could affect the best movements subset selection. We performed our experiments on EMG signals collected from four transradial amputees with an accuracy > $\mathbf{9 7 . 5 \%}$ on average across all subjects for the selection of best subset of movements.
\end{abstract}

\section{INTRODUCTION}

There have been many advances in multi-functional upper-limb prosthesis control, specifically in Pattern Recognition (PR) based EMG systems. Such systems offer intuitive control and the ability to control multiple movements in comparison with the conventional myoelectric control which offers a limited set of actions. Recently, PR systems have been deployed commercially for amputees' use [1], but their wide availability is still limited.

Each amputee has a unique set of characteristics related to his/her own amputation, e.g., different time since amputation, level of amputation, muscle structure left after amputation,

* Manuscript received February 22, 2016. The first author work is funded by the Ministry of Higher Education and Scientific Research, Iraq.

Ali H. Al-Timemy is with the Biomedical Engineering Department, AlKhwarzmi College of Engineering, University of Baghdad, Iraq. He is also a visiting research fellow at the Centre for Robotics and Neural Systems (CRNS), Cognitive Institute, Plymouth University, UK. Email: Ali.Ali@plymouth.ac.uk.

Rami Khushaba is within the Faculty of Engineering and Information Technology (FEIT), University of Technology, Sydney (UTS), 15 Broadway, Ultimo 2007, NSW, Australia. Email: Rami.Khushaba@uts.edu.au.

Javier Escudero * is with the Institute for Digital Communications, School of Engineering, The University of Edinburgh, Edinburgh, King's Buildings, EH9 3FB, United Kingdom. (Phone: +44 131650 5599, email: javier.escudero@ed.ac.uk).*Corresponding Author and the presence of nerve injury. Treating each amputee as an individual rather than grouping amputees together may be essential because each person might be able to perform some movements with a higher performance than other amputees. Therefore, each individual amputee's needs should be addressed by optimizing the movements with maximal performance. This would be a vital development for the future deployment of PR-based Electromyogram (EMG) controlled multi-functional upper-limb prosthesis. Having a reliable set of movements is important for achieving a usable system (error rates should be <10\%) [2]. Thus, finding the best movements that each individual amputee can perform is an important challenge.

Previous research investigated the selection of a usable subset of movements from a larger set classified with PR systems. Daley et al. [3] utilized high density EMG electrodes to determine a clinically acceptable number of electrodes with their locations for a reliable hand movements classification in transradial amputees. Time Domain (TD)Autoregressive Feature Extraction (FE) was used alongside Linear Discriminant analysis (LDA) classifier. It was found that four-to-six tasks could be classified with high accuracy of $86-95 \%$ for four recruited amputees [3]. In our previous work, a protocol was proposed for EMG site selection and movement assessment for the EMG based PR system [4]. The protocol found the best number of optimal movements and the best EMG channels from EMG data of two amputees. Time Domain FE and LDA classifier were used to perform the classification. It was found that every amputee has a specific set of optimal movements that can he/she can achieve differently from other amputees.

A method to select a subset of the hand gestures to maximize the sensitivity and specificity was proposed in [5]. EMG signals were collected from four intact-limbed subjects who performed ten finger and hand movements. Power spectral density average FE and LDA classifier were used with long analysis window size of 1 second. This scheme may be not suitable for PR since the delay may exceed the optimal controller delay [6]. Six gestures were chosen and these improved the sensitivity and specificity of the classifier using a proposed measure called positive-negative performance measurement index obtained by a series of confusion matrices. $\mathrm{Na}$ et al. [7] presented a personalized protocol to select usable movements for each of 20 intactlimbed subjects who performed 68 finger and wrist movements. A $k$-means clustering method was used to sort all movements into $k$ classes. Then, TD FE and artificial neural network classifier were used to perform the classification. 
It should be noted that the recent studies [5], [7] recruited only intact-limbed subjects for selecting the usable movement subset. All the previously reported studies used a PR system for selecting the best subset of hand/fingers movements based on a single feature extraction method and a single classifier.

In a different approach from previous work, this paper attempts to answer the following research question: 'Is the selection of the optimal number of movements for a given amputee different if the feature extraction or classifier is changed?' To answer this question, we collected EMG data from four transradial amputees and implemented a PR system with an overlapping windowing scheme, two FE methods and two classifiers. A series of experiments were then carried out to find the optimal number of movements for each individual amputee, with optimality here denoted by the number of movements that each amputee can perform across multiple trials with average error rates below $2.5 \%$.

\section{METHODOLOGY}

\section{A. Amputee subjects}

EMG signals were collected from the left stump of four transradial amputees, three acquired (TR1, TR2 and TR3) and one congenital amputee (CONG4), with left unilateral amputation. This data collection is part of larger study which investigated dexterous myoelectric control for transradial amputees. The EMG data for TR1-TR3 were collected at the Artificial Limbs and Rehabilitation Centers in Baghdad and Babylon, Iraq while EMG for CONG4 was collected at Plymouth University UK. The average age of the amputees was 26.5 years ( \pm standard deviation of 6.5 years). Stump length was TR1=13 cm, TR2 $=29 \mathrm{~cm}, T R 3=23 \mathrm{~cm}$ and CONG4 $=9 \mathrm{~cm}$ while time since amputations was TR $1=3$ years, TR2 $=27$ years and TR $3=7$ years. The amputees did not use a myoelectric prosthesis due to non-availability apart from CONG4 who used it for couple of years. Ethical approval was obtained from the Human Ethics Committee of the Faculty of Science and Technology at Plymouth University. All subjects were debriefed about the experiment and they gave their written informed consent to participate in the study.

\section{B. Experimental protocol and details of EMG signal acquisition}

Firstly, the stump was cleaned with alcohol and abrasive skin preparation gel (NuPrep®, D.O. Waver and Company, USA) was applied to the stump. Six pairs of $\mathrm{Ag} / \mathrm{AgCl}$ electrodes (Tyco healthcare, Germany), connected to a differential amplifier, were placed around the left stump. The ground reference electrode was placed on the Olecranon process of the Ulna. In Fig.1, an example of the locations of the electrodes on the stump of CONG4 amputee is shown.

EMG signals were acquired with a custom-built multichannel EMG amplifier (gain factor of 1000). The signals were sampled at a rate of $2000 \mathrm{~Hz}$ with 16-bit resolution data acquisition (USB-6210, National Instruments). For signal acquisition and display, LABVIEW software
(National Instruments, USA) was utilized. In this study, we investigated eight movement classes, including: 1) Thumb flexion, 2) Index flexion, 3) Fine pinch, 4) Tripod grip, 5) Spherical grip, 6) Pronation, 7) Supination and 8) no movement class (rest). These movements are categorized into 3 main groups a) Finger movements $(1,2)$, b) Grips patterns (3-5) and c) Wrist movements (6,7) (see table 1).

To collect the EMG signals from the four amputees, the following experimental protocol was used. First, the amputees were asked to imagine the given movement and produce a moderate force contraction with the stump and hold the position for 8-10 seconds for each imagined movement. We collected four trials of each movement. We utilized trials \#1 and \#2 in the training process while trials \#3 and \#4 were used in the testing process in order to evaluate the classification performance.

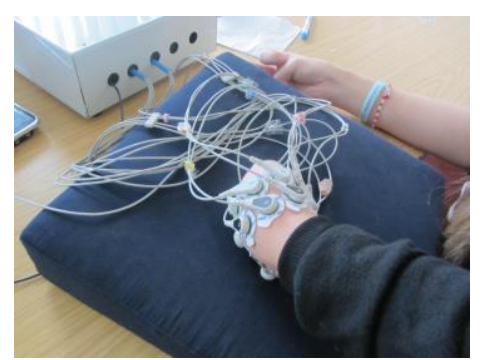

Figure 1. Electrode locations on the stump of the $4^{\text {th }}$ congenital amputee (CONG4).

\section{Patten recognition based EMG control}

In order to perform EMG pattern classification, we utilized an overlapped segmentation scheme with $150 \mathrm{~ms}$ window length and $50 \mathrm{~ms}$ window overlap. To investigate the effect of different feature extraction (FE) methods and classifiers on selecting the best subset of movement, we used two feature extraction methods and two classifiers. The first feature extraction method was the recently proposed Time Domain Power Spectral Descriptors (TD-PSD) [8], which showed an improvement in performance over the existing FE methods with fast processing time. In TD-PSD, a set of features describing the EMG power spectrum are extracted directly from the time-domain signal. Then, a cosine similarity function is employed to estimate the angle between the extracted power spectrum characteristics from the original EMG signals and their non-linear version. The resulting vector is then used as the final feature set [8].

The second FE method was the traditional Time Domain (TD) features [3], which consisted of integral absolute value, waveform length, number of zero crossings and number of slope sign changes. The total number of features (number of features $\times$ number of EMG channels) was 36 features for TDPSD FE and 24 features for TD FE. We then reduced the dimensionality of the extracted feature set with Spectral Regression (SR) dimensionality reduction, proposed by Cai et al. [9]. The SR method was also recently used in [8]. SR maps the original feature set into a new domain with $c-1$ features only, with $c$ being the number of classes, i.e., seven features in our problem.

To perform the classification of the reduced sets of features extracted in the previous step, we used two 
classifiers: Linear Discriminant Analysis (LDA) classifier [2] and Random Forest classifier (RF) [10]. The number of trees for RF classifier was 500 .

To sum up, four combinations of FE and classifiers will be investigated to find the best subset of movements, which are 1) TD-PSD+LDA 2) TD+LDA 3) TD-PSD+RF and 4) TD+RF. We used MATLAB ${ }^{\circledR}$ 2013a software (Mathworks, USA) to perform PR analysis in this study.

\section{Selecting the optimal subset of movements}

The main goal of this step is to find the best set of movements that each amputee can achieve with the lowest classification errors, defined as an acceptable level of error. In this study, we adopted the average error level of $(<2.5 \%)$ as a proof of concept.

We ran several iterations to find the optimal set of movements with each of the four FE/classifier combinations. In each iteration, we first perform the classification with all movements. Then, the classification errors are calculated for each movement from the confusion matrix for that given set of movements and the errors for all movements are examined individually. The movement with the highest level of error is identified and removed from the set of movements. This procedure is repeated until a set of movements with an average error below a predefined acceptable threshold (average error rate $<2.5$ ) is obtained.

\section{RESULTS AND DISCUSSION}

Fig.2 shows the classification errors, for all amputees, for different iterations of finding the best subset of movement for two FE methods (TD-PSD and TD) with LDA classifier while Fig. 3 shows the classification errors for all amputees with two FE methods (TD-PSD and TD) for Random Forest (RF) classifier. The error level of $2.5 \%$ is shown with a red dashed line.

The results shown in both Fig. 2 and Fig. 3 indicate few important points. Firstly, when a large number of movements is considered, the error rates achieved with the TD-PSD features were significantly lower than that achieved with the TD features ( $p$-value $<0.01)$ for both classifiers, which in turn agrees with the previous results in [8]. Secondly, Fig. 2 and Fig. 3 also show that the average number of iterations and the number of optimal movement classes were different for all amputees. This in turn further validate our hypothesis that an individual amputee would benefit from a PR-based system fitted based on their individual requirements rather than a unified system based on average overall performance. In terms of the reported results, to achieve an average error of less than $2.5 \%$, almost three iterations were required for TR1 and TR2 with TD-PSD while four-to-five iterations were needed for TR3 and CONG4.

An important advantage of identifying the best movement subset is that it identifies the movements with the lowest performance for each individual amputee. This can help the rehabilitation personnel (the occupational therapists) to perform the rehabilitation process on these movements and to apply a subject-specific movement rehabilitation scheme for the amputee.
The optimal number of movements that each amputee could produce with different classifier/FE is shown in Fig. 4. TR1 and TR2 amputees achieved the highest numbers of movement classes, while the TR3 and CONG4 achieved 4 movements. TR2 amputee has a long stump $(29 \mathrm{~cm})$ and there was no muscle deformation, which may be the reason why he achieved a better performance than other amputees.

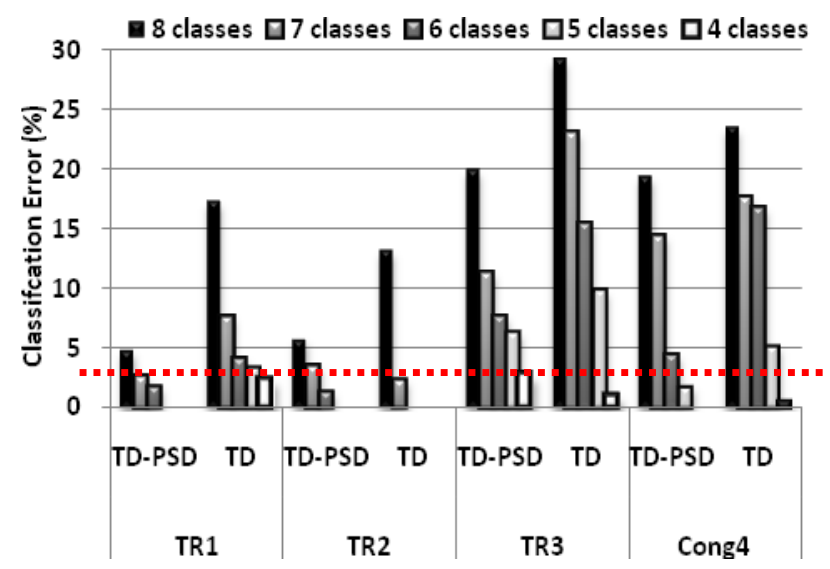

Figure 2. Average classification errors for each amputee with two FE methods (TD-PSD and TD) and LDA classifier. The error level of $2.5 \%$ is shown with a red dashed line.

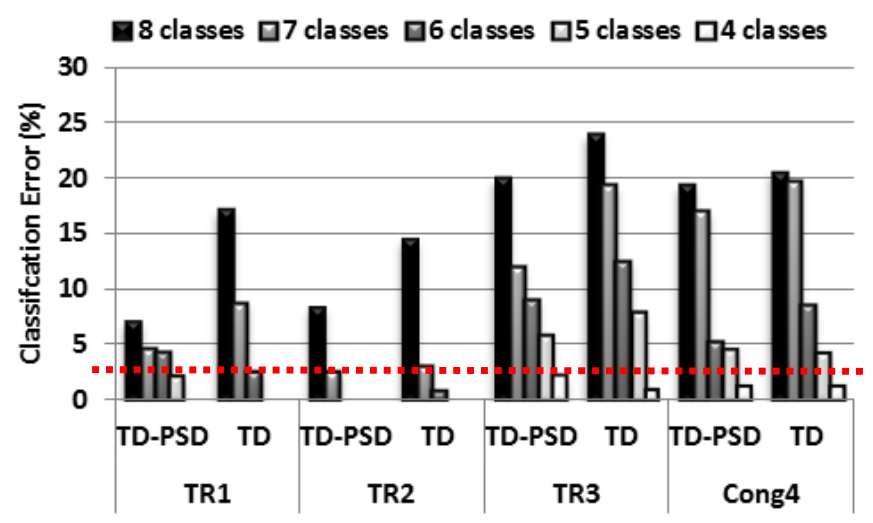

Figure 3. Average classification errors for each amputee with two FE methods (TD-PSD and TD) and RF classifier. The error level of $2.5 \%$ is shown with a red dashed line.

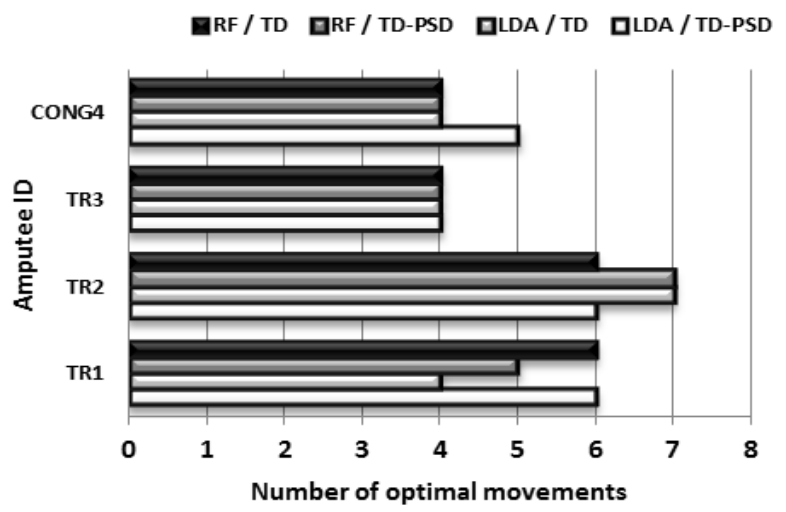

Figure 4. The optimal number of movements for each amputee with four combinations of classifier/FE with average classification error rate less than $2.5 \%$ 
A summary of the best movement subset with the four combinations of FE/classifier for each individual amputee is shown in Table I. From this table, it can be seen that the no movement class was clearly separated from all imagined movements for all amputees. In terms of the imagined movements classes with classification accuracy of $>97.5 \%$, pronation, supination and thumb flexion were among those highly separable from the remaining classes for all amputees, especially with the TD-PSD feature set. On the other hand, only TR2 was able to achieve high movement recognition rate for the index flexion movement, which was not the case for the remaining subjects. Finally, fine pinch and spherical grip movement had its highest recognition rates with TR1, TR2, and TR4 but not TR3. All of the above proves the importance of fitting a prosthesis system based on custom movements for each amputee to enhance the overall EMG pattern recognition performance.

TABLE I. The results of the best movement subset for four amputees with two FE methods (TD-PSD and TD) and 2 classifiers (LDA and RF). Best movements are shown in black for TR1, red for TR2, purple for TR3 and green for CONG4. The movement classes are: 1) Thumb flexion, 2) Index flexion, 3) Fine pinch, 4) Tripod Grip, 5) Spherical Grip, 6) Pronation , 7 ) Supination and 8) no movement class (rest).

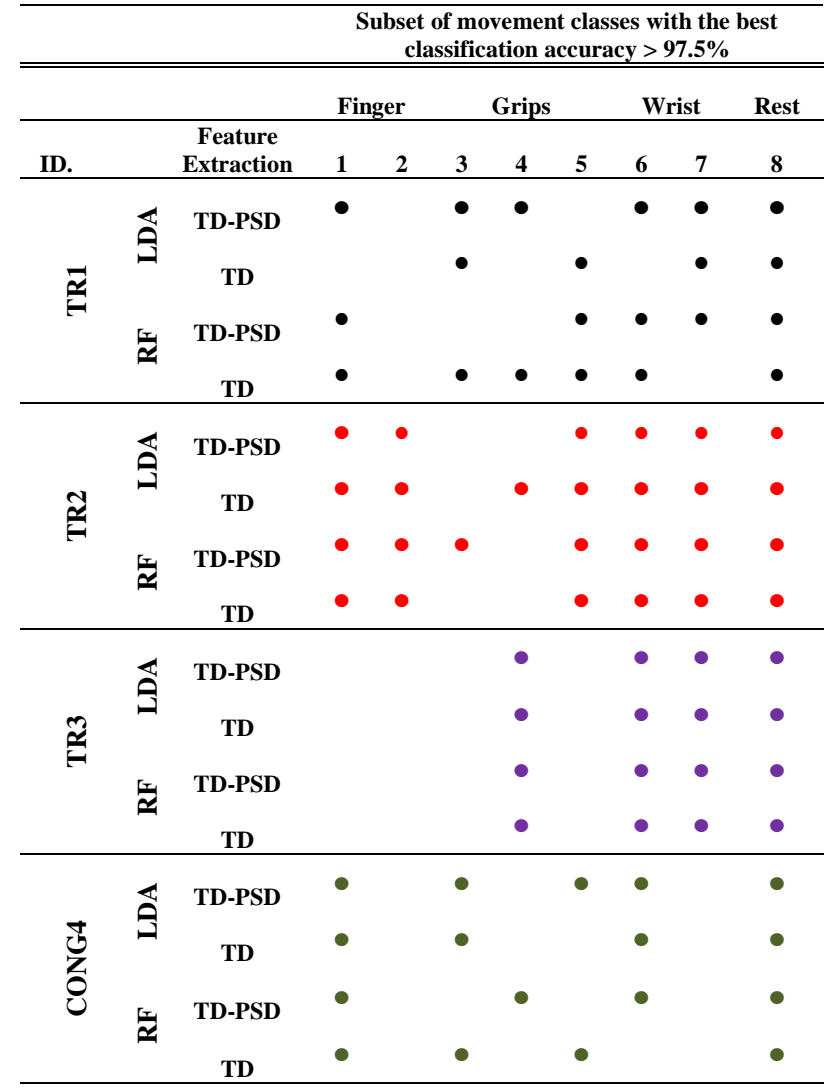

\section{CONCLUSION}

In this paper, we investigated the selection of a usable subset of hand/finger movements from a larger set classified with an EMG-based PR system. Unlike previous work focusing on individual feature extraction and classification methods, we performed experiments on data collected from four amputees while utilizing two time-domain based FE methods and two classifiers. Our experimental results showed significant reductions in terms of classification error rates when utilizing the TD-PSD features upon that of the TD features on large number of classes. The results also indicated a large variability among the amputees in terms of the optimal number of movements and the selection of these movements. As a result, tuning the parameters of EMG PR system based on individual needs can help optimize the system's performance based on the needs of each amputee.

\section{ACKNOWLEDGMENT}

The authors would like to thank Sarmad Haitham and Hussein Al-Jamal to help to recruit the amputees, Guido Bugmann for fruitful discussions, Hai $\mathrm{Li}$ and Nicholas Outram for building the EMG pre-amplifier and Dr. Adrain Chan for providing the Myoelectric Control Toolbox. We would like also to thank the amputees who participated in the study and the reviewers for their insightful comments.

\section{REFERENCES}

[1] A. L. Ciancio, F. Cordella, R. Barone, R. A. Romeo, A. D Bellingegni, R. Sacchetti, A. Davalli, G. Di Pino, F. Ranieri, V. Di Lazzaro, E. Guglielmelli, and L. Zollo, "Control of Prosthetic Hands via the Peripheral Nervous System," Front. Neurosci., vol. 10, p. 116 , Apr. 2016.

[2] E. Scheme and K. Englehart, "Electromyogram pattern recognition for control of powered upper-limb prostheses: state of the art and challenges for clinical use," J. Rehabil. Res. Dev., vol. 48, no. 6, pp. 643-659, 2011.

[3] H. Daley, K. Englehart, L. Hargrove, and U. Kuruganti, "High density electromyography data of normally limbed and transradial amputee subjects for multifunction prosthetic control," J. Electromyogr. Kinesiol., vol. 22, no. 3, pp. 478-484, 2012.

[4] A. H. Al-Timemy, J. Escudero, G. Bugmann, and N. Outram, "Protocol for Site Selection and Movement Assessment for the Myoelectric Control of a Multi-Functional Upper-Limb Prosthesis," The 35th Annual International Conference of the IEEE Engineering in Medicine and Biology Society (EMBC). Osaka, Japan, 2013.

[5] M. C. F. Castro, S. P. Arjunan, and D. K. Kumar, "Selection of suitable hand gestures for reliable myoelectric human computer interface," Biomed. Eng. Online, vol. 14, no. 30, pp. 1-11, 2015.

[6] T. R. Farrell, "Determining delay created by multifunctional prosthesis controllers," J. Rehabil. Res. Dev., vol. 48, no. 6, pp. xxixxxvii, 2011.

[7] Y. Na, S. Kim, S. Min, C. Choi, S. Kim, S. Jo, and J. Kim, "Personalized Protocol to Select Usable Movements for Myoelectric Pattern Recognition," in 6th European Conference of the International Federation for Medical and Biological Engineering SE - 8, vol. 45, I. Lacković and D. Vasic, Eds. Springer International Publishing, 2015, pp. 29-33.

[8] A. H. Al-Timemy, R. N. Khushaba, G. Bugmann, and J. Escudero, "Improving the Performance against Force Variation of EMG Controlled Multifunctional Upper-Limb Prostheses for Transradial Amputees," IEEE Trans. Neural Syst. Rehabil. Eng., 2016.

[9] D. Cai, X. He, and J. Han, "SRDA: An Efficient Algorithm for LargeScale Discriminant Analysis," IEEE Trans. Knowl. Data Eng., vol. 20, no. 1, pp. 1-12, 2008.

[10] L. Breiman, "Random Forests," Mach. Learn., vol. 45, no. 1, pp. 532, Oct. 2001. 